\begin{tabular}{|c|c|}
\hline Title & Optical pulse compression to 5.0 fs by use of only a spatial light modul ator for phase compensation \\
\hline Author(s) & Karasawa, Naoki; Li, Liming; Suguro, A kira; Shigekawa, Hidemi; Morita, Ryuji; Y amashita, Mikio \\
\hline Citation & $\begin{array}{l}\text { Journal of the Optical Society of A merica B : O ptical Physics, 18(11), 1742-1746 } \\
\text { https://doi.org/10.1364/JOSA B.18.001742 }\end{array}$ \\
\hline Issue Date & 2001-11-01 \\
\hline Doc URL & http:/hdl.handle.net/2115/45331 \\
\hline Rights & C 2001 Optical Society of A merica \\
\hline Type & article \\
\hline File Information & JOSAB18-11_1742-1746.pdf \\
\hline
\end{tabular}

Instructions for use 


\title{
Optical pulse compression to 5.0 fs by use of only a spatial light modulator for phase compensation
}

\author{
Naoki Karasawa \\ Department of Applied Physics, Hokkaido University, and Core Research for Evolutional Science and Technology, \\ Japan Science and Technology Corporation, Kita-13, Nishi-8, Kita-Ku, Sapporo, 060-8628 Japan \\ Liming Li \\ Department of Photonics Material Science, Chitose Institute of Science and Technology, and Core Research for \\ Evolutional Science and Technology, Japan Science and Technology Corporation, 758-65 Bibi, Chitose, \\ 066-8655 Japan
}

Akira Suguro

Citizen Watch Co., Ltd., 840 Shimotomi, Tokorozawa, 359-8511 Japan

Hidemi Shigekawa

Institute of Applied Physics, University of Tsukuba, and Core Research for Evolutional Science and Technology, Japan Science and Technology Corporation, 1-1-1 Tennodai, Tsukuba, 305-8573 Japan

\section{Ryuji Morita and Mikio Yamashita}

Department of Applied Physics, Hokkaido University, and Core Research for Evolutional Science and Technology, Japan Science and Technology Corporation, Kita-13, Nishi-8, Kita-Ku, Sapporo, 060-8628 Japan

Received March 13, 2001; revised manuscript received May 8, 2001

We experimentally demonstrate the generation of 5.0 -fs optical pulses $(2.5 \mu \mathrm{J}, 1-\mathrm{kHz}$ repetition rate), using only a spatial light modulator for phase compensation. Pulse compression of the broadband pulse (500-1000 $\mathrm{nm}$ ) from an argon-filled capillary fiber is achieved with a liquid-crystal spatial light modulator without any prechirp compensation. The output pulse width is found to be $4.1 \mathrm{fs}$ by a fringe-resolved autocorrelator fitted with a transform-limited pulse and to be $5.0 \mathrm{fs}$ by second-harmonic generation frequency-resolved optical gating with marginal correction. It is to our knowledge the shortest pulse ever generated by use of only a spatial light modulator for phase compensation. (c) 2001 Optical Society of America

OCIS codes: $320.7090,320.5560,320.7140,230.6120,320.5520,190.7110$.

\section{INTRODUCTION}

Optical pulses of $\sim 5$ fs have been generated directly from an oscillator ${ }^{1,2}$ and external compression by use of a fused-silica fiber, ${ }^{3-5}$ a capillary fiber filled with a noble gas, ${ }^{6,7}$ or optical parametric amplification ${ }^{8}$ for spectrum broadening. All these methods utilize chirped mirrors for chirp compensation. Chirped mirrors have the advantage of high throughput. However, difficulty in obtaining the large bandwidth, interdependence of different phase orders, and an inability to fine-tune the phase in the experimental setup are disadvantages. A pulse shaping technique ${ }^{9}$ that uses a liquid-crystal spatial light modulator (SLM) for pulse compression has the advantages of large bandwidth (300-1500 nm) and in situ adaptive phase control. Chirped pulses from an oscillator have been compressed from 80 to 11 fs by a SLM with a two-dimensional search algorithm for second- and thirdorder dispersion coefficients. ${ }^{10}$ Recently the SLM was used to compress the broadband pulses from an argon- filled capillary fiber with prechirp compensation by a prism pair to yield 6 -fs pulses ${ }^{11}$ and sub-6-fs pulses. ${ }^{12}$ Deformable mirrors were used as phase-only modulators to compress pulses from 92 to $15 \mathrm{fs}^{13}$ In this paper we demonstrate that a SLM can be used to compress broadband (500-1000-nm) pulses from an argon-filled capillary fiber, without any prechirp compensation, to generate 5.0-fs pulses. When prechirp compensation optics such as a prism pair is not used, the optical throughput increases, and alignment becomes easier. To evaluate accurately the pulses that are thus generated, we used not only a fringe-resolved autocorrelator (FRAC) but also a second-harmonic generation ( $\mathrm{SH})$ frequency-resolved optical gating (FROG) apparatus. ${ }^{4,5,7}$

\section{EXPERIMENTAL SETUP}

The experimental setup is shown in Fig. 1. The output beam of a Ti:sapphire laser-amplifier system [Femto- 
power Pro; center wavelength, $790 \mathrm{~nm}$; pulse width, $30 \mathrm{fs}$; repetition rate, $1 \mathrm{kHz}$; pulse energy adjusted by a neutraldensity filter (ND), $140 \mu \mathrm{J}$ ] was focused into a capillary fiber with 34-cm length and $0.1-\mathrm{mm}$ inner diameter, which was positioned in a chamber filled with argon. ${ }^{14}$ The chamber had two 1-mm-thick sapphire windows. The output beam from the chamber was collimated by a spherical mirror and was directed to a $4-f$ system by the SLM. The 4- $f$ system consisted of two spherical mirrors with a focal length $f=20 \mathrm{~cm}$ and two silver reflective gratings (G1 and G2) with a ruling distance $d$ $=1 / 150 \mathrm{~mm}$. The optical path lengths from the gratings to the spherical mirrors and those from the spherical mirrors to the SLM were all set to be $f$. The liquid crystal SLM (Citizen Company) consisted of 648 97- $\mu \mathrm{m}$-wide pixels, with a $5-\mu \mathrm{m}$ gap between adjacent pixels. The transmission of the SLM was more than $70 \%$ at a $500-700-\mathrm{m}$ wavelength but decreased at long wavelengths (e.g., $50 \%$ at $1000 \mathrm{~nm}$ ). The pulse energy after the fiber chamber was $22 \mu \mathrm{J}$, and the throughput of the 4- $f$ system was $30 \%$. In the setup we used periscopes (PSs) to change the height, the polarization direction, or both of the beam, and a flip mirror (FM) to change the beam direction for measurements of the FRAC and the SH FROG.

\section{DETERMINATION OF THE SLM PHASE PATTERN}

The SLM was used as a phase modulator, which can impose a phase variation of $2 \pi$ for light whose wavelength is less than $1400 \mathrm{~nm}$ with a resolution of 192. The Phase $\phi_{\mathrm{SLM}}(x)$ from the SLM at position $x$ is given by a polynomial of the form

$$
\begin{aligned}
\phi_{\mathrm{SLM}}(x)= & \frac{\alpha}{2}\left[\omega(x)-\omega_{0}\right]^{2}+\frac{\beta}{6}\left[\omega(x)-\omega_{0}\right]^{3} \\
& +\frac{\gamma}{24}\left[\omega(x)-\omega_{0}\right]^{4},
\end{aligned}
$$

where $\omega_{0}$ is the center angular frequency for a Taylor expansion, $\omega(x)$ is the angular frequency at position $x$ and is given by $\omega(x)=2 \pi c /\left\{d \sin \left[\tan ^{-1}(x / f)+\sin ^{-1}\left(\lambda_{c} / d\right)\right]\right\}, c$ is the speed of light, and $\lambda_{c}=800 \mathrm{~nm}$ is the wavelength at the central position of the SLM, $x=0 . \quad \lambda_{c}$ does not have to be equal to the center wavelength for Taylor expansion $\lambda_{0}$, where $\lambda_{0}=2 \pi c / \omega_{0} . \quad \alpha, \beta$, and $\gamma$ are the group-delay dispersion (gdd), the third-order dis-

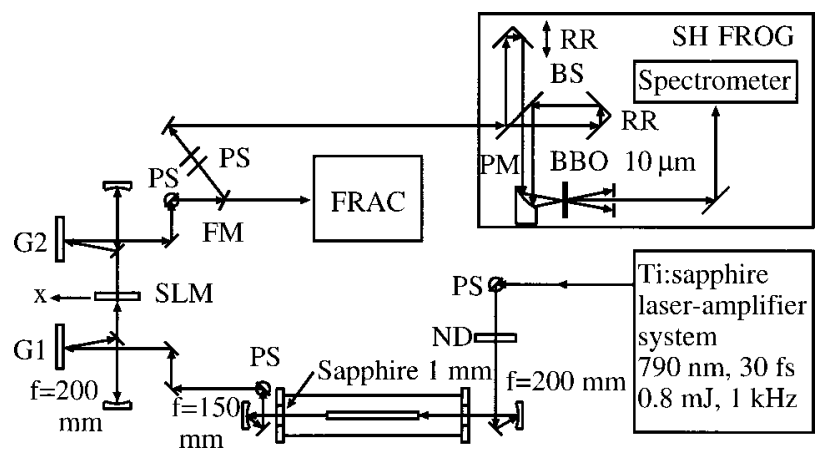

Fig. 1. Experimental setup for generation of 5.0-fs optical pluses. See text for definitions.

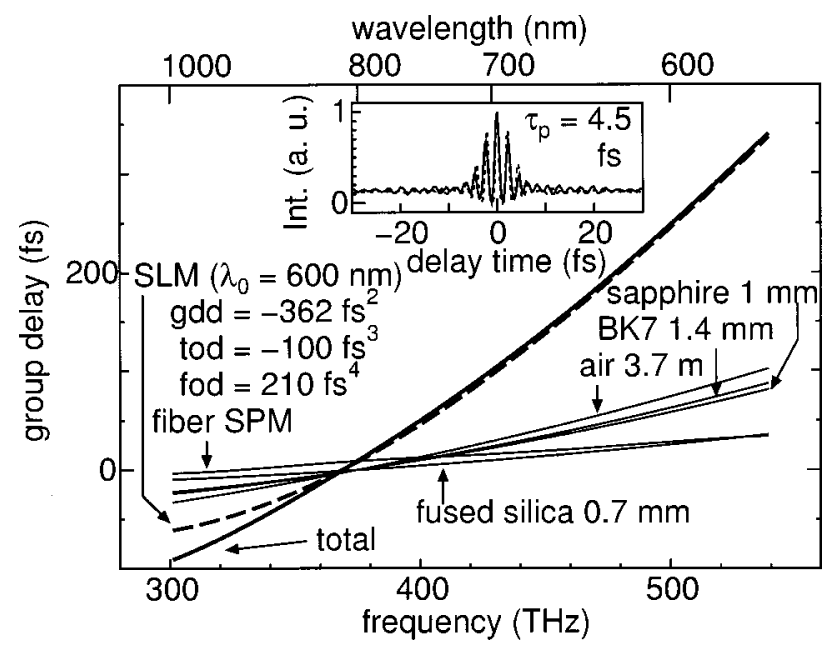

Fig. 2. Calculated group delay of each optical component (solid curves) at $\lambda_{0}=600 \mathrm{~nm}$ at an argon pressure of $2.0 \mathrm{~atm}$. The total group delay (thick solid curve) is compared with the negative of the experimentally optimized group delay applied by the SLM (dashed curve). Inset, experimental (solid curve) and 4.5-fs fitted transform-limited (dotted curve) FRAC traces at these parameters.

persion (tod), and the fourth-order dispersion (fod), respectively, at $\omega_{0}$. The group delay $t_{d, \mathrm{SLM}}(x)$ imposed by the SLM was

$$
\begin{aligned}
& t_{d, \mathrm{SLM}}(x)= \frac{\partial \phi_{\mathrm{SLM}}(\omega)}{\partial \omega} \\
&=\alpha\left[\omega(x)-\omega_{0}\right]+\frac{\beta}{2}\left[\omega(x)-\omega_{0}\right]^{2} \\
& \\
&+\frac{\gamma}{6}\left[\omega(x)-\omega_{0}\right]^{3} .
\end{aligned}
$$

Parameters $\alpha, \beta$, and $\gamma$ were initially estimated from the total group delay $t_{d}(\omega)$ of the optical components from a capillary fiber (including self-phase modulation) to a nonlinear crystal in the measuring apparatus. $t_{d}(\omega)$ was fitted in the form of Eq. (2), and $\alpha, \beta$, and $\gamma$ were obtained. Then the negative values of these fitted parameters were initially imposed by the SLM to satisfy $t_{d, \mathrm{SLM}}(\omega)$ $+t_{d}(\omega) \simeq$ constant in the whole frequency range. Theoretically, this condition should produce the shortest pulses. However, in practice it was necessary to adjust the phase determined by the SLM to yield the shortest pulses because of the difference in group delay between calculations and experiments, which were due mainly to the approximations used in our calculations. Use of the Taylor expansion in Eq. (1) was found to be the natural way to perform fine adjustment of the phase. Parameters $\alpha, \beta$, and $\gamma$ were fine-tuned by a computer to yield the shortest pulse with the FRAC and later with the $\mathrm{SH}$ FROG apparatus. In Fig. 2 the group delay of each component in the optical path and its negative value obtained with the SLM, as well as the totals of these values, are shown for which the shortest FRAC trace (Fig. 2, inset, the 4.5-fs fitted transform-limited pulse) was obtained for conditions when $\lambda_{0}$ and the argon pressure were set to $600 \mathrm{~nm}$ and $2.0 \mathrm{~atm}$, respectively. As shown in Section 5 below, FROG results for 5.0-fs pulses were obtained under 
these conditions. Here, the effective group delay that arises from propagation in the capillary fiber was obtained from numerical nonlinear propagation calculations. ${ }^{14}$ For other optical components, we calculated the group delay by using the Sellmeier equations for fused silica (beam splitters of FRAC-FROG, $0.7 \mathrm{~mm}$ ), ${ }^{15}$ BK7 glass (SLM substrates, $1.4 \mathrm{~mm}){ }^{16}$ sapphire (a chamber window, $1 \mathrm{~mm}),{ }^{17}$ and air $(3.7 \mathrm{~m}) .{ }^{18}$ As shown in Fig. 2 , the agreement between the calculated group delay (thick solid curve) and the negative of the group delay from the SLM (dashed curve) when the parameters were optimized is reasonably good.

\section{EFFECTS OF FINITE PIXEL SIZE OF THE SLM}

$\lambda_{0}$ in Eq. (1) was initially set to be $800 \mathrm{~nm}$ with values of $\alpha, \beta$, and $\gamma$ different from those given in Section 3; the FRAC trace shown in Fig. 3, which was fitted well for the 4.1-fs transform-limited pulse, was obtained at an argon pressure of $2.8 \mathrm{~atm}$. However, in this case the pulse width measured by the FROG ( $\sim 6 \mathrm{fs}$ ) was longer than the transform-limited value. This underestimate of the pulse width may be understandable as having resulted from the poor fit of the pedestals in the autocorrelation trace $^{19}$ and the asymmetric temporal intensity profile as well as the slightly different dispersion optics in the FRAC and the FROG apparatuses. Thus we were motivated to search for better parameters for the SLM. Because of the finite pixel size, the phase imposed by the SLM in Eq. (1) was stepwise, and, as the difference in phase between adjacent pixels [which we call $\Delta \phi(\lambda)$ ] became larger, phase compensation became more difficult because of the phase error introduced by the finiteness of the pixel width. For our experimental setup the frequency width for each pixel became larger as the wave-

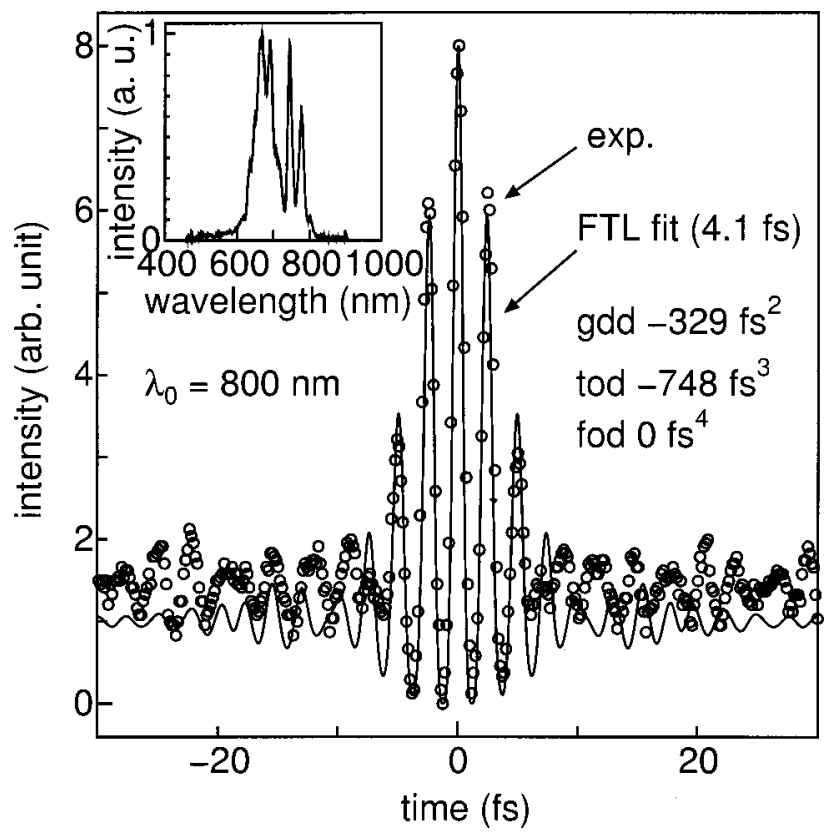

Fig. 3. Experimental (circles) and fitted transform-limited (FTL, solid curve) interferometric autocorrelation traces obtained at $\lambda_{0}=800 \mathrm{~nm}$ at an argon pressure of $2.8 \mathrm{~atm}$. Inset, the pulse spectrum.
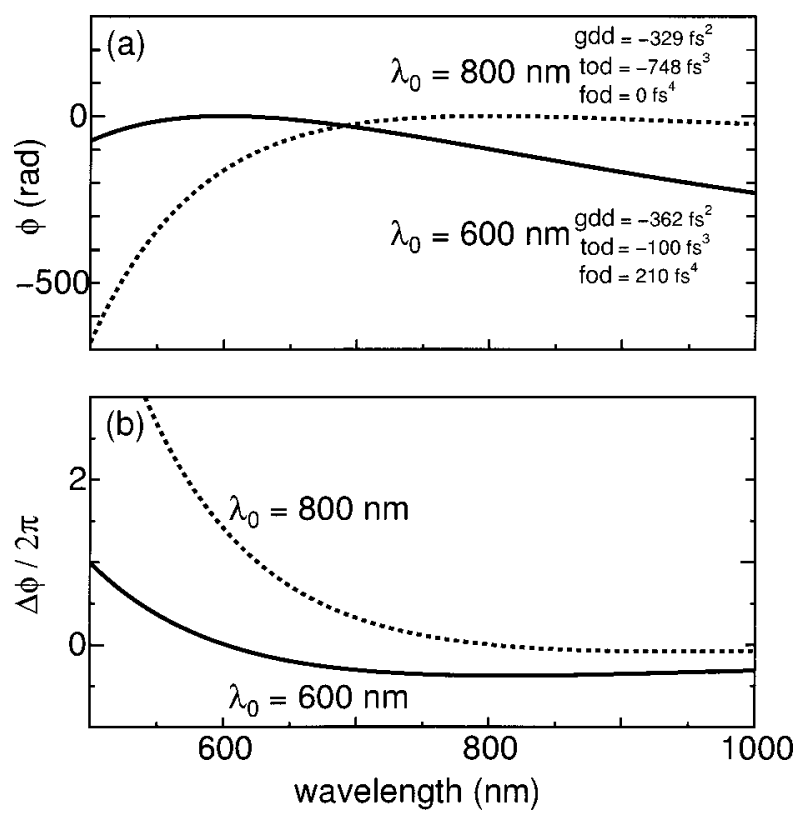

Fig. 4. (a) Applied phase $\phi(\lambda)$ and (b) phase change per pixel $\Delta \phi(\lambda)$ of the SLM for $\lambda_{0}=600 \mathrm{~nm}$ (solid curves; parameters used in Fig. 2) and $\lambda_{0}=800 \mathrm{~nm}$ (dotted curves; parameters used in Fig. 3).

length became shorter because of the almost-linear relationship between the pixel number and the wavelength of the SLM. Thus $|\Delta \phi(\lambda)|$ tended to become larger as the wavelength became shorter than $\lambda_{0}$. In Fig. 4 the applied phase and the phase variation per pixel at the SLM for $\lambda_{0}=600 \mathrm{~nm}$ (Fig. 2) and $\lambda_{0}=800 \mathrm{~nm}$ (Fig. 3) are shown. In both cases the fitted group delays $t_{d, \mathrm{SLM}}(\omega)$ were almost identical. However, the fitted phases were quite different, as shown in this figure. We analyzed $|\Delta \phi(\lambda)|$ of the SLM and found that for $\lambda_{0}=800 \mathrm{~nm}$ it exceeded $\pi$ for wavelengths below $672 \mathrm{~nm}$; it was difficult to achieve phase compensation below this wavelength. This effect was pronounced because the spectrum that was generated had a peak at $670 \mathrm{~nm}$ owing to the low transmission of light in the infrared region at the SLM. When $\lambda_{0}=600 \mathrm{~nm},|\Delta \phi(\lambda)|$ in the shorter-wavelength range decreased considerably, and it exceeded $\pi$ only for the wavelength below $540 \mathrm{~nm}$, yielding significantly better phase compensation; pulses much closer to the transform limit were obtained with the FROG measurements.

\section{SH FROG RESULTS}

In Fig. 5 the results of pulse measurements with the $\mathrm{SH}$ FROG at $\lambda_{0}=600 \mathrm{~nm}$ are shown. Owing to the slight difference in the optics used in the FRAC and in the FROG apparatuses, we readjusted the SLM parameters slightly for the FROG measurements. In the SH FROG apparatus (Fig. 1) a 0.5-mm-thick broadband (400-1300$\mathrm{nm}$ ) dielectric beam splitter (BS) was used to separate the beam that was directed to the silver-coated retroreflector (RR) in a balanced configuration. Beam separation $d_{b}$ at the parabolic mirror (PM) was $2 \mathrm{~mm}$; beam diameter $w_{m}$ was $1 \mathrm{~mm}$. The time smearing ${ }^{4}$ was $\tau_{d}$ $=\sqrt{2 \ln 2} \lambda d_{b} /\left(\pi c w_{m}\right)=1.6 \mathrm{fs} \quad$ at wavelength $\lambda$ $=650 \mathrm{~nm}$, with Gaussian space and time profiles as- 


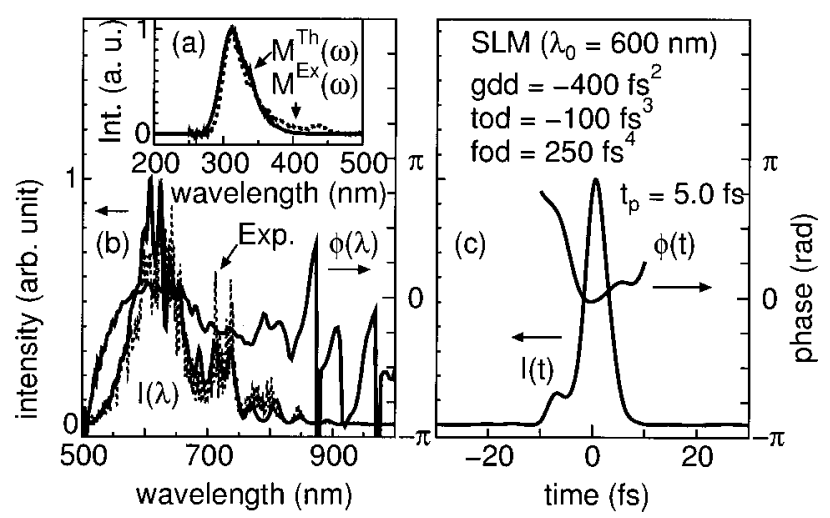

Fig. 5. Results of the corrected frequency marginal corrected FROG measurements with SLM parameters. (a) Margin calculated from the experimental spectrum $M^{\mathrm{Th}}(\omega)$ (solid curve) and that obtained from the FROG trace $M^{\mathrm{Ex}}(\omega)$ (dotted curve). (b) Spectrum intensity $I(\lambda)$ and phase $\phi(\lambda)$. Dotted curves experimental spectrum. (c) Temporal intensity $I(t)$ and phase $\phi(t)$.

sumed. The $10-\mu \mathrm{m}$-thick $\beta$-barium borate crystal (BBO; Fig. 1) at a cutting angle of $40^{\circ}$ was used in type 1 geometry. Owing to the limited bandwidth of the crystal, the frequency marginal ${ }^{20}$ calculated from the FROG trace $M^{\mathrm{Ex}}(\omega)$ and that from the spectrum intensity autoconvolution $M^{\mathrm{Th}}(\omega)$ did not match, as can be seen from in Fig. 5(a). To correct for this effect we multiplied each value in the FROG trace by a frequency-dependent factor such that the marginal calculated from the FROG trace became identical to that obtained from the spectrum. ${ }^{20}$ After correction of the marginal we used commercial FROG software (Femtosoft) to retrieve the pulse intensity and phase. In the measurements, a 1024-channel optical multichannel analyzer with an intensified CCD was used. The step number was 256 and the delay time was $1.34 \mathrm{fs}$. The time required for the measurement was $\sim 1 \mathrm{~min}$, and the stability of the pulses was excellent during that time. Also, we could usually reproduce the pulse width measured by FROG within $10 \%$ by using the same parameters several hours after they had been optimized. In Figs. 5(b) and 5(c) the spectrum and the temporal waveforms, respectively, of the pulse are shown. The FROG error was 0.0038 . The measured and the calculated spectra agree quite well because of the correction of the marginals. However, the temporal width was not changed by that correction. The $5.0-\mathrm{fs}$ pulse obtained had 2.4 cycles at the center wavelength $(633 \mathrm{~nm})$, and its width was $14 \%$ longer than the transform-limited pulse width (4.4 fs for the FROG measurement). If the geometrical smearing effect is taken into account, we estimate that the pulse width will be $4.7 \mathrm{fs}$. The residual phase was within $\pi / 2$ in the wavelength range $(550-800 \mathrm{~nm})$, where the spectral intensity is significantly large.

\section{CONCLUSION}

SLM-only phase compensation to generate a 5.0-fs pulse has been experimentally demonstrated. It has been shown that the choice of the center wavelength for a Taylor expansion of the phase obtained from a SLM is important for obtaining pulses that are near the transform limit. The pulse width evaluated by correction of the frequency marginal of a 5.0-fs SH FROG is to our knowledge the shortest pulse width ever generated by use of a SLM as the only phase compensator.

\section{ACKNOWLEDGMENT}

The authors thank K. Oka for accurate determination of the phase-change characteristics of the SLM.

N. Karasawa's e-mail address is n-karasa@ photon.chitose.ac.jp. Present address, Department of Applied Photonics System Technology, Chitose Institute of Science and Technology, 758-65 Bibi, Chitose 066-8655 Japan.

\section{REFERENCES}

1. U. Morgner, F. X. Kärtner, S. H. Cho, Y. Chen, H. A. Haus, J. G. Fujimoto, E. P. Ippen, V. Scheuer, G. Angelow, and T. Tschudi, "Sub-two-cycle pulses from a Kerr-lens modelocked Ti:sapphire laser," Opt. Lett. 24, 411-413 (1999).

2. D. H. Sutter, G. Steinmeyer, L. Gallmann, N. Matuschek, F. Morier-Genoud, U. Keller, V. Scheuer, G. Angelow, and T. Tschudi, "Semiconductor saturable-absorber mirrorassisted Kerr-lens mode-locked Ti:sapphire laser producing pulses in the two-cycle regime," Opt. Lett. 24, 631-633 (1999).

3. A. Baltuška, Z. Wei, M. S. Pshenichnikov, D. A. Wiersma, and R. Szipöcs, "All-solid-state cavity-dumped sub-5-fs laser,” Appl. Phys. B 65, 175-188 (1997).

4. A. Baltuška, M. S. Pshenichinikov, and D. A. Wiersma, "Amplitude and phase characterization of 4.5-fs pulses by frequency-resolved optical gating," Opt. Lett. 23, 14741476 (1998).

5. A. Baltuška, M. S. Pshenichinikov, and D. A. Wiersma, "Second-harmonic generation frequency-resolved optical gating in the single-cycle regime," IEEE J. Quantum Electron. 35, 459-478 (1999).

6. M. Nisoli, S. Stagira, S. De Silvestri, O. Svelto, S. Sartania, Z. Cheng, M. Lenzner, Ch. Spielmann, and F. Krausz, "A novel high-energy pulse compression system: generation of multigigawatt sub-5-fs pulses," Appl. Phys. B 65, 189196 (1997).

7. Z. Cheng, A. Fürbach, S. Sartania, M. Lenzner, Ch. Spielmann, and F. Krausz, "Amplitude and chirp characterization of high-power laser pulses in the 5-fs regime," Opt. Lett. 24, 247-249 (1999).

8. A. Shirakawa, I. Sakane, M. Takasaka, and T. Kobayashi, "Sub-5-fs visible pulse generation by pulse-front-matched noncollinear optical parametric amplification," Appl. Phys. Lett. 74, 2268-2270 (1999).

9. A. M. Weiner, D. E. Leaird, J. S. Patel, and J. R. Wullert II, "Programmable shaping of femtosecond optical pulses by use of 128-element liquid crystal phase modulator," IEEE J. Quantum Electron. 28, 908-919 (1992).

10. D. Yelin, D. Meshulach, and Y. Silberberg, "Adaptive femtosecond pulse compression," Opt. Lett. 22, 1793-1795 (1997).

11. L. Xu, N. Nakagawa, R. Morita, H. Shigekawa, and M. Yamashita, "Programmable chirp compensation for 6-fs pulse generation with a prism-pair-formed pulse shaper," IEEE J. Quantum Electron. 36, 893-899 (2000).

12. L. Xu, L. Li, N. Nakagawa, R. Morita, and M. Yamashita, "Application of a spatial light modulator for programmable optical pulse compression to the sub-6-fs regime," IEEE Photonics Technol. Lett. 12, 1540-1542 (2000).

13. E. Zeek, K. Maginnis, S. Backus, U. Russek, M. Murnane, G. Mourou, H. Kapteyn, and G. Vdovin, "Pulse compression by use of deformable mirrors," Opt. Lett. 24, 493-495 (1999). 
14. N. Karasawa, R. Morita, H. Shigekawa, and M. Yamashita, "Generation of intense ultrabroadband optical pulses by induced phase modulation in an argon-filled single-mode hollow waveguide," Opt. Lett. 25, 183-185 (2000).

15. I. H. Malitson, "Interspecimen comparison of the refractive index of fused silica," J. Opt. Soc. Am. 55, 1205-1209 (1965).

16. Schott Glaswerke, Schott optical glass catalog (Schott Glaswerke, Mainz, Germany, 1992)

17. I. H. Malitson, "Refraction and dispersion of synthetic sapphire,” J. Opt. Soc. Am. 52, 1377-1379 (1962).
18. D. E. Gray, ed., American Institute of Physics Handbook, 3rd ed. (McGraw-Hill, New York, 1972).

19. L. Gallmann, D. H. Sutter, N. Matuschek, G. Steinmeyer, and U. Keller, "Techniques for the characterization of sub10-fs optical pulses: a comparison," Appl. Phys. B 70, S67S75 (2000).

20. G. Taft, A. Rundquist, M. M. Murnane, I. P. Christov, H. C. Kapteyn, K. W. DeLong, D. N. Fittinghoff, M. A. Krumbügel, J. N. Sweetser, and R. Trebino, "Measurement of 10-fs laser pulses," IEEE J. Sel. Top. Quantum Electron. 2, 575585 (1996). 